\title{
Communicating and Interpreting Wearable Sensor Data with Health Coaches
}

\author{
Kimiko Ryokai ${ }^{1}$, Florian Michahelles ${ }^{2}$, Mareike Kritzler ${ }^{2}$, Suhaib Syed ${ }^{1}$ \\ ${ }^{1}$ School of Information \\ University of California, Berkeley \\ Berkeley, California. USA \\ $\{$ kimiko, susyed\}@berkeley.edu \\ ${ }^{2}$ Siemens Corporation \\ 2087 Addison Street, 2nd floor \\ Berkeley, California. USA \\ \{florian.michahelles, mareike.kritzler\}@siemens.com
}

\begin{abstract}
We present a pilot study, which investigates the role of activity data visualization in the context of interaction and communication with health coaches. The data is obtained from commercially available wearable wellness sensors such as Basis Watch, or Lumo Back. We studied how interface design's ability to synthesize and flexibly display data from wearable sensors help participants to understand their daily wellness related physical condition and activities. We also investigated how the interface enhances the communication between users and their health coaches. We discuss the implications for the design.
\end{abstract}

Keywords-Wearable sensors; wellness; health coaches; data visualization

\section{INTRODUCTION}

In the commercial sector, a large number of wearable wellness sensors are available today (e.g., Fit Bit, Basis Watch, Lumo Back, etc. See Vandrico webpage [6] for a current list). Some early adopters of such technologies wear several of these sensors to continuously tack their daily activities and body conditions. Yet there is a lack of tools that support the interpretation of large amount of data collected by these devices. It is up to the users to come up with their own workaround solutions to interact with and interpret data from a variety of apps. Another emerging question is whether any of the data collected by consumer level, non-FDA approved devices that users collect would be useful for health care professionals in interacting with their patients. If so, in what ways can the interface design contribute to interpreting the data? Can interface tools for health activity data empower everyday users to feel in control of their own data while at the same time help the health care professional in providing their care? We set out to investigate these research questions by designing and evaluating HealthViz, an interactive visualization tool that pulls together data from several commercially available sensors as well as smart phone applications and support both the users of these technologies and their health care providers. We present our iterative process of designing HealthViz informed by interviews and design sessions with medical and health care providers. We also report results from a preliminary pilot study with five individual users who wore these devices to collect their activity data for one week and shared their data with a health coach using the HealthViz system. We discuss the implications for the design of user interfaces in supporting interpretation of health and wellness data between everyday users and health coaches.

\section{RELATED WORK AND REVIEW OF CONSUMER LEVEL WEARABLE SENSORS}

Much research effort has been made on designing data visualization from personal health trackers for interpretation, as well as studying if such data will be useful towards facilitating collaboration between health professionals and patients/users [e.g., 1,2,3,5]. Our work builds on this effort but focuses on integration of data from commercially available wearables, and study the potential of UI design, which is driven by the needs of health coaches more directly.

We began our research by reviewing both currently available and forthcoming wearable sensors, both commercial products and academic research prototypes. The goal was to develop a landscape that covers a variety of existing and emerging sensing technologies. As resources, web search, blogs, crowd-funding platforms, as well as quantified-self communities were included. This informed the team both what devices are available for the current study as well as foreseeable trend in the future. While many of the sensors were still Kickstarter campaigns, we identified two commercial sensors we were immediately able to work with: Basis Watch and Lumo Back in combination with the Moves mobile application as a unique combination to provide users with basic wellness data combined with activity types and geo-location data associated with such data.

\section{INTERVIEWS WITH MEDICAL PROFESSIONALS}

Next, in order to identify what type of information collected by consumer level sensors could be useful for what type of patient scenarios, we conducted semi-structured interviews with two medical doctors and two nurse practitioners. While it was difficult to identify specific scenarios given the variety of medical needs for different diseases, a couple of illness agonistic themes emerged in reference to possible uses and capabilities of commercially available wellness sensors:

First, medical doctors and nurses have decidedly limited time they could spend with their patients. Yet, up to about a third of their time is used in information-seeking, i.e., gathering the patients' medical history, their daily habits, goals that pertain to the current medical condition. The rest of the time is used in medical decision-making (diagnosing) and information- 
giving (patient education) (see also Ong et al. [4]). Medical doctors and nurses explained that people are not always good at reporting their past events and patterns of their activities, and therefore, they saw potential of these sensors in shortening information-seeking time. The doctors explained that even if the sensors are not FDA approved devices, having access to general trend lines of patients' heart rate that go over an extended period of time, for example, could be useful in determining whether the questionable sample in the data is an anomaly or consistent pattern that persist over time.

Second, in the information-giving phase (educating the patients about how to stay healthy), doctors and nurses expressed the importance of relating to patients' life styles and habits, and identifying individual goals that motivate the patients to actually make necessary changes in their behaviors to stay healthy. Therefore, the health goals have to be manageable and realistic for each patient; otherwise it is difficult for the patients to follow them. In referencing data with patients, as one doctor said, we have to present them with "actionable data." Doctors thought that tools they can share with patients to see their everyday habits and patterns could be useful in identifying and agreeing on such actionable data unique to each patient.

From these interviews, we also confirmed that medical doctors' availabilities are extremely limited not only in interacting with patients, but also with possible design researchers. Therefore, as our first collaborators in this initial phase of our research, we decided to work with professional health coaches. Professional health coaches work with patients in improving and maintaining their health both within and outside the medical environment. Health coaches are more available to spend time with patients and provide knowledge that bridges the medical domain and everyday life and activities of patients.

\section{Design Sessions with Health Coaches}

In synthesizing our findings on commercially available sensors and interviews with medical and health care providers, we identified a set of possible design components for our system. We explored UI design components with measurements we could reasonably acquire from commercial devices over time, i.e., heart rate, calories, steps, perspiration, air and skin temperatures, activity types (walking, running, bicycling, and taking transportation), and location data. We then conducted two 90-minute design sessions with two health coaches to further refine the UI design. We recruited two health coaches who have training in medical settings. As a design task, we asked the health coaches to arrange the paper mockup windows of different data visualizations so that they fit their needs and form ideal configurations in interacting with their patients.

In coming up with an ideal UI configuration, both health coaches wanted to have heart rate as the most important data to track and visualize over time. Both coaches wanted to have a large space dedicated for detailed visualizations of heart rate patterns. Others such as calories, steps, temperatures, and posture data were auxiliary for both coaches, and they could be made visible when necessary. Similarly, health coaches found geo-location data to be useful, but it could stay "on the side." From these observations, we modified our HealthViz UI accordingly, establishing visual hierarchy to support health coach's needs in interpreting data with their patients.

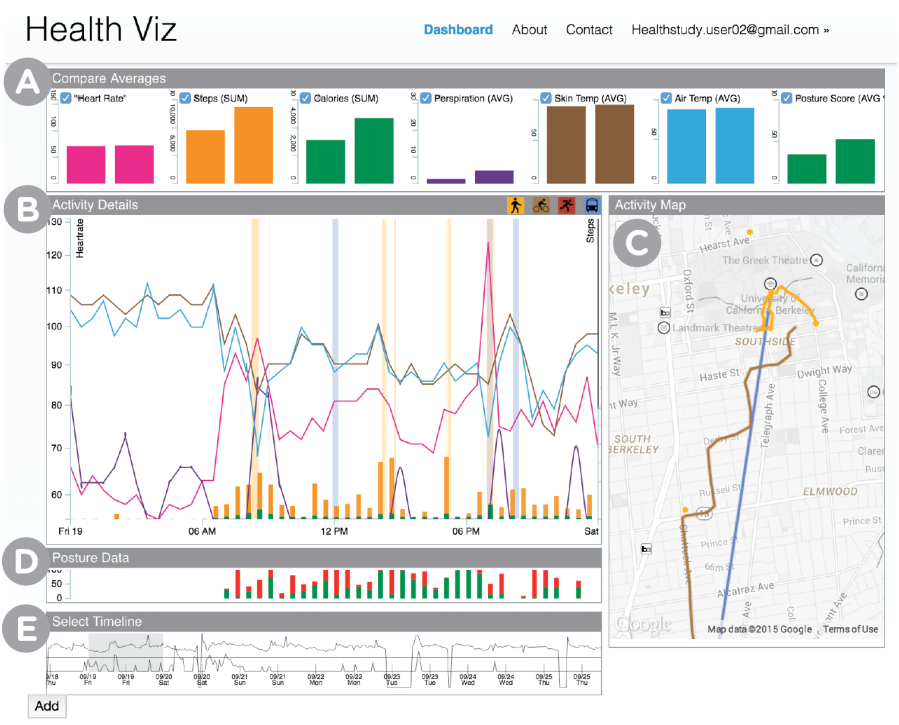

Figure 1. A) Average values of each biometrics for selected period. B) Main window visualizes the biometrics data (heart rate, steps, calories, perspiration, temperature) all at once or separately. The window could be zoomed to reveal the details. C) Dynamic map visualization with color-coded paths (e.g., orange is for running, brown is for bicycling, blue for transportation). D) Posture data visualization (green is holding good posture and red is not). E) Timeline selection. User can brush over to select a particular period to visualize.

\section{HealthViz System}

HealthViz is an interactive visualization tool that currently pulls together data from the Basis Watch, Lumo Back, and Moves app. It allows for a more holistic view of data to support both users and health coaches (Figure 1). In the main window (Fig. 1B) HealthViz visualizes the biometric data from a Basis Watch. Each biometric data type can be displayed individually, or together with other types. Users can select a particular period (e.g., a single day or multiple days) to visualize by brushing over the timeline selection (Fig.1E). Users can compare the averages of their biometric data (e.g., steps during the weekdays vs. weekend) using our compare tool (Fig.1A). The map (Fig.1C) dynamically shows the location data (i.e., zooms into relevant locations) based on the selected timeline and show color-coded paths (bicycling, walking, running, etc.).

The HealthViz system is made up of three modules: storage, controller and the visualization module. The storage module retains the user details and the data from different health sensors (Basis/ Lumo Back/ Moves) for all the users. It is implemented using a MYSQL database. This data is collected and processed by the controller module. It downloads the data from different sources every six hours and then stores it in the database. HealthViz is a multi-user system and these user profiles and their authentications are managed by this layer. It is also responsible for sharing this data with the Visualization module when a user logs in. The controller module is implemented in PHP and Python and relies on some external libraries for downloading the sensor data from the Basis Servers. The dashboard appears after logging in, it is 
implemented in the visualization module. It loads the data by firing a sequence of HTTP GET requests and plots it using the D3.js and Google Maps javascript library.

\section{HEALthViz Pilot STUdy}

We conducted a pilot study to evaluate our initial design and to observe how HealthViz supports the communication between patients and health coaches as well as the understanding of the patient's activities.

Participants: Five individuals, two female and three male, ages 25 to 38 participated in our study. Two participants were working professionals and three were graduate students. While all participants showed interests in the technology, none of the five participants have worn commercially available wellness sensors prior to the study. One of the two health coaches from the design sessions participated as the health coach in this pilot study.

Method: The participants were informed that the purpose of the study was to evaluate the performance of the information visualization system, not their performance. They were asked to wear the Basis Watch, the Lumo Back belt, and their smartphone running the Moves app every day, as long as they would feel comfortable, for one week. During this one week, participants were asked to have three 30-minute meetings: a first meeting (on Day 1) to setup the devices and be informed about the study procedure; a second meeting in the midway through the study (on Day 4) with a health coach who will help to interpret the data visualization; and a third meeting at the end of the week (on Day 8) with an experimenter to discuss their experience. The participants were also asked to look at the activity data through HealthViz at least once per day.

\section{FINDINGS}

Despite the demand of having to wear multiple devices on their body every day for one week (and having to remember to charge and sync all these devices), all participants (except one who decided not to wear Lumo Back after the 3rd day because of its difficulty synching up with his phone) wore all the devices consistently for all 8 days. On the 8 th day, all five participants indicated that they would continue to wear a Basis Watch beyond the study if they had the chance, but not Lumo Back. All participants met with a health coach on Day 4 and shared their activity data using HealthViz. All participants continued wearing the devices and interacted with HealthViz until the Day 8 when they had the final interview with a researcher. Many of the discussions between the health coach and participants were initiated by them looking together at the peaks and valleys of the participants' heart rate in HealthViz. In the following sections, we discuss four emerging roles that our HealthViz played in supporting communication between health coach and participants as well as the interpretation of health data by the health coach.

\section{A. Memory Aid with Concrete References}

The HealthViz system provided opportunities for health coach and participants to engage in discussions regarding concrete instances of their health related activities and opportunity for advice. For example, David and the health coach identified David's abnormal pattern of heart rate:

Coach: "What's happening here? [pointing at one of the highest heart rate peaks on the HealthViz]?"

David: "I'm pretty sure that's when I was out drinking."

David knew that he had reaction to alcohol but he did not know how those events with alcohol compared to other peaks of his heart rate if this had not been visualized in HealthViz with a health coach pointing him to this.

In the post study interview, the health coach explained that, "People tend to be poor historians of their own events and behaviors. So, health coaches typically have to spend a lot of time gathering that information. This is valuable time that could be spent on talking to patients about actual changes. This way (with HealthViz), I get a very fair picture of what it already is, getting into it. And more importantly, I know it's accurate." The UI can play a key role in providing such concrete reference points to launch discussions.

\section{B. Provide the Context, Longer View, and Whole Picture}

Being able to see the details of concrete instances of their activities was important. But also important was the ability to access the "whole picture" for identifying recurring patterns that point to participants' habits, and to contrast the two perspectives.

Alex: "This (HealthViz) provides a longer view, the whole picture. By looking at this (visualization), I can see I'm this kind of person. I wake up and exercise. On weekends, I go on hikes (pointing at the map). Looking at it all together in one place, I get the whole picture of who I am, which is missing from health apps today."

By looking at the map, Russell and Chloe noticed that their activities are concentrated in their small local area, but because of their professions, it was difficult for them to go out of their way to engage in additional exercise. This became an opportunity for the health coach to jump in and suggest a manageable goal for each of them: "As you find yourself walking, try engaging in brisk walks to raise your heart rate just a little bit more."

Many current health applications focus on showing details of biometrics and do not provide this type of long-term view combining data from diverse sources for users to access a holistic view of their activities. This ability to access a bigger picture, i.e., which biometric data relate to which human activities, seems to make the interaction with health data more manageable.

For the health coach, this was useful information to provide meaningful and personal advice. For example, the coach said, "If I saw that a person is going to a farmer's market regularly, that gives additional information about this person's interests in cooking or eating healthy." On the other hand, for the participants, this also raised the issue of privacy. While none of the participants objected to sharing information with a health coach during one week, three of the five participants in the post study interview expressed that the UI should eventually have a feature where users can decide to reveal or hide certain information, especially the location data. While the issue of 
privacy is beyond the scope of this paper, we plan to carefully study it in our future work, taking into account the tension between sharing and privacy and the UI's ability to give control to the users.

\section{Accessing Expert's Perspectives}

HealthViz provided a place where participants could gain access to experts' perspective on how to interpret the data. The meetings with a health coach seemed to change the way participants interacted with HealthViz subsequently:

Lucy: "I learned that the patterns of peaks and valleys of heart rate is more important than how high or low the heart rate was independent of its context."

Russell: "I probably knew it, or I probably could have looked it up. But being able to learn it in the context of sharing my real data with a health coach made it meaningful."

In subsequent interactions with HealthViz on her own, Chloe felt that the visualization was "connected to a person (health coach) who helped translate it." This shows that there is an opportunity for the UI design to both moderate experts' view and serve as an active lens through which people engage with and interpret their health data.

\section{Active Participation from Both Sides}

In sharing access to HealthViz, participants seeing what experts see (notice or question) was important, but experts seeing what patients see (what they are wondering about) was equally important. HealthViz provided a space where this sharing of perspectives could occur. In the post interview with a health coach, the health coach explained, "A lot of people tend to disengage (during health coach sessions) because it is a passive experience for them. Information is going from the health care provider to the person. It is not an active experience, but with this (HealthViz), we have a shared tool that we are both using, and I'm getting the information from the participant or patients that's filling the blanks for me, and with that very information for me, that is filling the blanks for them, how to contextualize the data, how to personalize, give meaning to the data. Because it has that back and forth, it makes the 30 minutes so much more valuable."

\section{E. Limitations and Future Work}

The current pilot study is limited in terms of its scale. The number of participants is low and the participants were all healthy individuals, highly motivated and excited about new technology. In future work, we plan to conduct a long-term study, which goes over multiple weeks and include a wider range of participants.

While participants enjoyed interacting with HealthViz over one week, all of them mentioned that the interface could be improved to be more intuitive. Some participants preferred to have a larger map in the center, which focuses on a summary of their movements first and then reveals biometrics associated with the movements. Some participants wanted to have a summary of their overall achievements (e.g., "Did I break my own record for the number of steps last week?") independent of locations. Yet other participants wanted to have an even longer view in the center, for example showing a whole month of data with different trend lines. This points to a possible solution where users can customize the visual hierarchy of the screen by defining which windows should take over the main area and which should remain peripheral.

We are also working to include annotation capability for both users and health coach. For the health coach, beyond their face-to-face sessions, this allows for more direct ways to leave recommendations or reminders as direct part of the visualization. For users, this allows for more direct ways to annotate and mark questionable data to be discussed with a health coach.

\section{DISCUSSIONS AND CONCLUSION}

We have presented our iterative process of designing and evaluating HealthViz. In our preliminary study, we looked at how people made sense of their activity data collected with consumer level sensors, and how health coaches can help them interpret the data in more personally meaningful ways. Our preliminary results showed that the HealthViz was useful for both the health coach and everyday users in effectively sharing their perspectives while minimizing information-gathering time, thus allowing for more time in making personal recommendations and goal setting. With more wearable health sensors and devices on the horizon, it is important to consider how the interfaces support everyday users' interpretation of data with experts' view and in their personal context, which can lead to real health benefits.

While preliminary, this work shows the potential for such UI design research. Design of HCI projects related to health and wellness may do well by not just focusing on details of biometric data and users' individual engagement with such data, but also to consider the role the UI could play in supporting communication between health care professionals and everyday users.

\section{ACKNOWLEDGMENTS}

We would like to thank Shubham Goel, Shaun Giudici, and our study participants. Special thanks goes to Steven DeMello, Hema Doshi, Dr. Dinesen, Dr. Han, Dr. Miyamoto, Dr. Bair, and Basis Watch.

\section{REFERENCES}

[1] Bagalkot, N. \& Sokoler, T. 2011. MagicMirror: towards enhancing collaborative rehabilitation practices. In Proc. of CSCW. ACM, NY. 593-596.

[2] Boutrup, J., Odelheim, K., Sokoler, T., Bagalkot, N.L. 2013. MyReDiary: exploring the design for supporting adherence to physical rehabilitation. In Proc. of PervasiveHealth '13. ICST, Brussels, 284-287.

[3] Li, I., Dey, A.K., Forlizzi, J. 2012. Using context to reveal factors that affect physical activity. ACM Trans. Comput.-Hum. Interact. 19, 1, 7.

[4] Ong LM, de Haes JC, Hoos AM, Lammes FB. Doctor-patient communication: a review of the literature. Soc Sci Med. 1995, 40(7):903-18.

[5] Yan,K., Tracie, B., Marie-Ève, M., Mélanie, H., Jean-Luc, B., Benoit, T. St-Onge, M., Marie, L. 2014. Innovation through Wearable Sensors to Collect Real-Life Data among Pediatric Patients with Cardiometabolic Risk Factors. International Journal of Pediatrics. Vol. 2014. Hindawi Publishing.

[6] Vandrico Wearable Technology Database http://vandrico.com/database 